\title{
Differential Expressions of Apoptosis-related Genes in Lung Cancer Cell Lines Determine the Responsiveness to lonizing Radiation
}

\author{
Su-Yeon Lee ${ }^{1}$, Moon-Kyung Choi ${ }^{2}$, Jung-Min \\ $\mathrm{Lim}^{2}$, Hong-Gyun $\mathrm{Wu}^{3,4}$, Ju $\mathrm{Han} \mathrm{Kim}^{1}$ and \\ Woong-Yang Park ${ }^{2 *}$
}

${ }^{1}$ Seoul National University Bioinformatics, ${ }^{2}$ Departments of Biochemistry and Molecular Biology, and ${ }^{3}$ Radiation Oncology, ${ }^{4}$ Cancer Research Institute, Seoul National University College of Medicine, Seoul 110-799, Korea

\begin{abstract}
Radiotherapy would be the choice of treatment for human cancers, because of high cost-effectiveness. However, a certain population of patients shows a resistance to radiotherapy and recurrence. In an effort to increase the efficacy of radiotherapy, many efforts were driven to find the genes causing the unresponsiveness to ionizing radiation. In this paper, we compared the gene expression profiles of two lung cancer cell lines, H460 and H1299, which showed differential responses to ionizing radiations. Each cell were irradiated at 2 Gy, and harvested after $0,2,4,8,12$ and 24 hours to examine the expressions. Two-way ANOVA analysis on time-series experiments of two cells could select 2863 genes differentially expressed upon ionizing radiation among 32,321 genes in microarray $(p<0.05)$. We classified these genes into 21 clusters by SOM clustering according to the interaction between cell types and time. Two SOM clusters were enriched with apoptosis-related genes in pathway analysis. One cluster contained higher levels of phosphatidyl inositol 3-phosphate kinase (PI3K) subunits in $\mathrm{H} 1299$, radio-resistant cells than $\mathrm{H} 460$, radiosensitive cells. TRAIL receptors were expressed in $\mathrm{H} 460$ cells while the decoy receptor for TRAIL was expressed in H1299 cells. From these results, we could characterize the differential responsiveness to ionizing radiation according to their differential expressions of apoptosis-related genes, which might be the candidates to increase the power of radiotherapy.
\end{abstract}

Keywords: apoptosis, ionizing radiation, lung cancer, radio-sensitivity, radiotherapy

*Corresponding author: E-mail wypark@snu.ac.kr

Tel +82-2-740-8241, Fax +82-2-744-4534

Accepted 2 March 2008

\section{Introduction}

Radiotherapy would play an important role in cancer treatment for long times and widely used to have more than one million patients received radiotherapy every year (Wu et al., 2002). In some type of cancers at prostate and cervix, radiotherapy achieved high performance in cure rate in comparison to radical surgery. Moreover, it can be applied to organ conservation surgery in breast cancers and rectal cancers. However, a certain proportion of patients do not respond to radiotherapy, and the mechanism of radiation resistance has been the main target of research to increase the efficacy of radiotherapy.

Although single molecules were targeted to modulate the radiation responses at pre-clinical models, it was hard to be applied to patients. It might be due to the complexity of radiation responses as shown in gene expression profiling experiments (Park et al., 2002). Recently miRNA also has been proposed to be important regulator of radiation responses (Weidhaas et al., 2007). Gene expression profiling using microarray provided valuable tools for the clinical oncology to determine the prognosis of patients (Lossos et al., 2004; Pomeroy et al., 2002), the molecular diagnosis (Golub et al., 1999) as well as the responsiveness to therapeutics (Snyder and Morgan, 2004).

There have been many reports on the molecular pattern analysis using microarray to understand the chemoand radio-resistance in cervical cancer (Achary et al., 2000; Tewari et al., 2005; Wong et al., 2006), rectal cancer (Kim et al., 2007) and esophageal cancer (Fukuda et al., 2004). Most of the studies are to identify differentially expressed genes in patients with different clinical outcomes, which can be applied to the evaluation of prognosis more accurately. Although the conventional parameters like tumor stage and grade can be used to decide optimal cancer therapy, molecular markers would provide valuable information to make clinical decisions (Klopp and Eifel, 2006). Genome-wide analysis on gene expression can predict the clinical consequences more accurately. In addition, the information from gene expression profiling can facilitate the development of biological target for therapeutics by identifying pathways and determining steps contributing to the phenotype.

In this study, we examined the expression profiles of two lung cancer cell lines, which showed differential re- 
sponses to ionizing radiation. Especially the time-series data of two cell lines revealed radiation response-related genes from constitutively up- or down-regulated genes in two cells. Especially we focused on the apoptosis pathway in different clusters of radiation response to explain the differential responses upon ionizing radiations.

\section{Methods}

\section{Cell culture and ionizing radiation}

H460 and H1299 lung cancer cells were purchased from ATCC and maintained in high-glucose Dulbecco's Eagle's medium (DMEM; GIBCO/BRL, Gaithersburg, Md., USA) containing $10 \%$ heat-inactivated fetal bovine serum (FBS, GIBCO/BRL) and $50 \mathrm{U} / \mathrm{ml}$ gamma-interferon (Gemzyme, Cambridge, MA, USA).
After the exposure to ionizing radiation generated by 4MV linear accelerator (Clinac 4/100, Varian, Palo Alto, $\mathrm{CA})$, cells were harvested at the indicated time. We repeated sets of experiment three times to collect biological triplicates in every sample.

\section{Microarray and data analysis}

Samples in each group were harvested in triplicates and total RNAs were extracted by dissolving in TriZol and the purification using Qiagen RNAeasy column (Park et al., 2002). We used GeneChip Human Gene 1.0 ST array from Affymetrix which includes 32,000 human genes. Fluorescence intensity was processed and measured using Exon Microarray Analyzer. Intensity data were imported to an in-house microarray database as described previously (Lee et al., 2006).

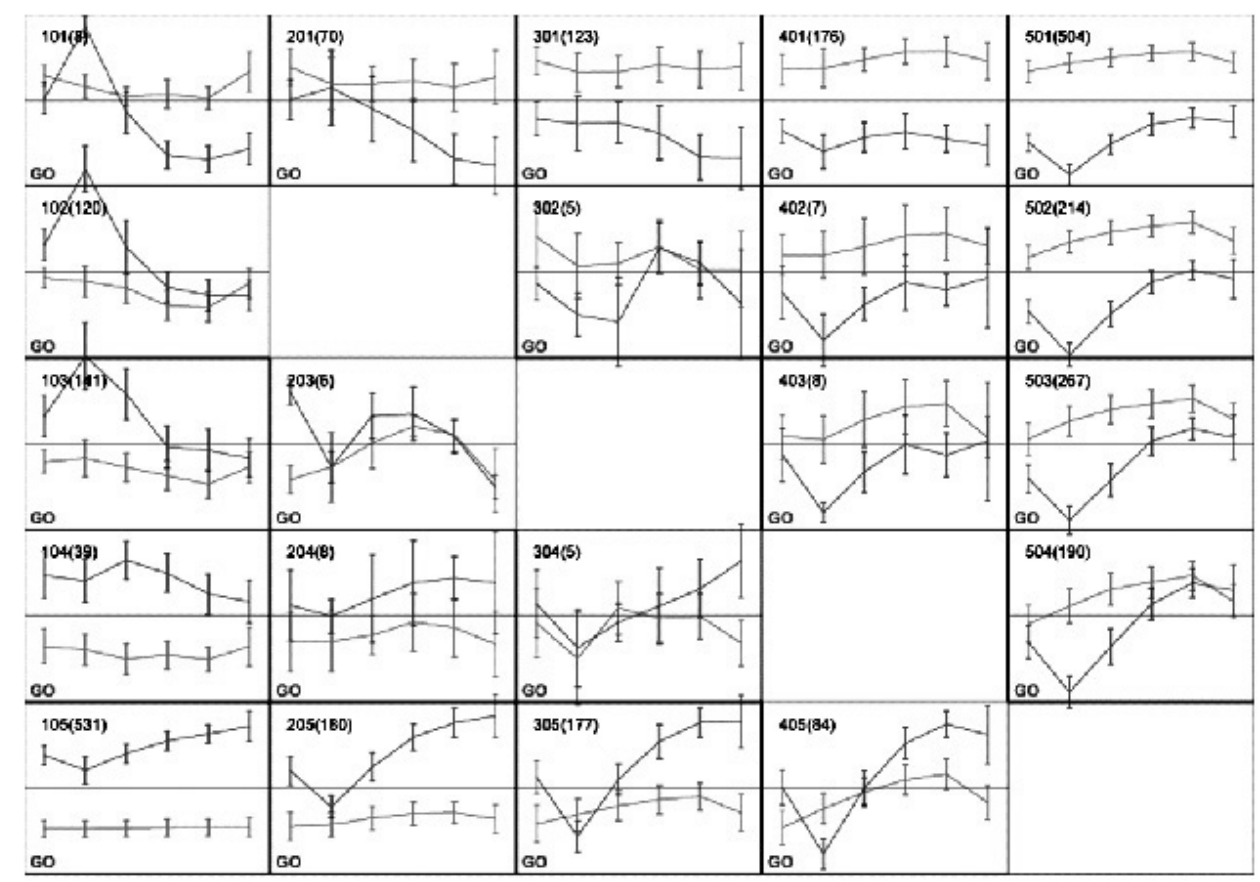

Fig. 1. SOM cluster analysis on 2863 differentially expressed genes.

Table 1. Classification of SOM clusters according to their response patterns upon ionizing radiation

\begin{tabular}{cll}
\hline \multicolumn{1}{c}{ Group } & Response pattern* & \multicolumn{1}{c}{ Cluster (gene No.) } \\
\hline Radio-sensitive (RS), & Constitutive (RSC) & 104 (39), $105(531)$ \\
higher in radio-sensitive H460 cells & Early (RSE) & $102(120), 103(141)$ \\
(H460>H1299) & Late (RSL) & $205(180), 305(177), 405(84)$ \\
Radio-resistant (RR), & Constitutive (RRC) & $301(123), 401(176)$ \\
higher in radio-resistant H1299 cells & Early (RRE) & $501(504), 502(214), 503(267), 504(190)$ \\
$(\mathrm{H} 1299>\mathrm{H} 460)$ & Late (RRL) & $201(70)$ \\
\hline
\end{tabular}

\footnotetext{
*"Response pattern" represents the patterns of transcriptional regulation in $\mathrm{H} 460$ cells upon ionizing radiation.
} 
38 Genomics \& Informatics Vol. 6(1) 36-43, March 2008

\section{Gene Ontology (GO) and pathway analysis}

Differentially expressed genes were further analyzed using DAVID for GO analysis as well as pathway analysis (Dennis et al., 2003).

\section{Results and Discussion}

To examine the molecular changes upon ionizing radiation, we used H460 and H1299 lung cancer cells, which show different responses to ionizing radiation in the clo-

Table 2. Gene ontology (GO) analysis on the genes in RS clusters

\begin{tabular}{|c|c|c|c|c|c|c|}
\hline $\begin{array}{l}\text { Response } \\
\text { pattern }\end{array}$ & $\begin{array}{c}\text { Cluster } \\
\text { (gene No.) }\end{array}$ & GO term & Count & $\%$ & $p$ value & $\begin{array}{l}\text { Fold } \\
\text { change }\end{array}$ \\
\hline \multirow[t]{6}{*}{ RSE } & \multirow[t]{3}{*}{$102(120)$} & Signal transduction & 27 & 25.00 & 0.00362 & 1.703 \\
\hline & & Inflammatory response & 6 & 5.56 & 0.00368 & 5.746 \\
\hline & & Response to biotic stimulus & 14 & 12.96 & 0.01004 & 2.162 \\
\hline & \multirow[t]{3}{*}{$103(141)$} & G-protein coupled receptor protein signaling pathway & 17 & 13.49 & 0.00093 & 2.504 \\
\hline & & Response to stimulus & 28 & 22.22 & 0.00485 & 1.678 \\
\hline & & Phosphoinositide-mediated signaling & 4 & 3.17 & 0.01234 & 8.234 \\
\hline \multirow[t]{12}{*}{ RSC } & \multirow[t]{2}{*}{$104(39)$} & Regulation of progression through cell cycle & 5 & 13.16 & 0.00731 & 6.096 \\
\hline & & Regulation of physiological process & 11 & 28.95 & 0.04775 & 1.808 \\
\hline & \multirow[t]{10}{*}{105} & Regulation of cell proliferation & 23 & 4.60 & 4.3E-06 & 3.146 \\
\hline & & Humoral immune response & 13 & 2.60 & 0.00069 & 3.246 \\
\hline & & Enzyme linked receptor protein signaling pathway & 13 & 2.60 & 0.00203 & 2.866 \\
\hline & & Cell adhesion & 30 & 6.00 & 0.00232 & 1.815 \\
\hline & & Apoptosis & 26 & 5.20 & 0.00258 & 1.907 \\
\hline & & Cell differentiation & 22 & 4.40 & 0.01247 & 1.781 \\
\hline & & Response to stress & 38 & 7.60 & 0.02858 & 1.414 \\
\hline & & Dephosphorylation & 9 & 1.80 & 0.02973 & 2.470 \\
\hline & & Amino acid and derivative metabolism & 14 & 2.80 & 0.03143 & 1.921 \\
\hline & & Secretion & 12 & 2.40 & 0.03187 & 2.064 \\
\hline \multirow[t]{24}{*}{$\mathrm{RSL}$} & \multirow[t]{9}{*}{$205(180)$} & Catabolism & 18 & 10.40 & $2.4 \mathrm{E}-05$ & 3.329 \\
\hline & & Cellular lipid metabolism & 15 & 8.67 & $6.9 \mathrm{E}-05$ & 3.584 \\
\hline & & Cellular carbohydrate metabolism & 10 & 5.78 & 0.00323 & 3.298 \\
\hline & & Phospholipid biosynthesis & 4 & 2.31 & 0.01089 & 8.635 \\
\hline & & Positive regulation of protein kinase activity & 4 & 2.31 & 0.01317 & 8.050 \\
\hline & & Protein biosynthesis & 14 & 8.09 & 0.01934 & 2.030 \\
\hline & & Glycoprotein metabolism & 5 & 2.89 & 0.03631 & 3.984 \\
\hline & & Cytokine biosynthesis & 3 & 1.73 & 0.04197 & 9.134 \\
\hline & & Response to stress & 16 & 9.25 & 0.04482 & 1.705 \\
\hline & \multirow[t]{10}{*}{$305(177)$} & Vesicle-mediated transport & 9 & 5.17 & 0.01121 & 2.943 \\
\hline & & Transport & 35 & 20.11 & 0.01181 & 1.488 \\
\hline & & Small GTPase mediated signal transduction & 7 & 4.02 & 0.02899 & 2.995 \\
\hline & & Regulation of cell proliferation & 7 & 4.02 & 0.03906 & 2.787 \\
\hline & & Protein transport & 10 & 5.75 & 0.04377 & 2.132 \\
\hline & & Wnt receptor signaling pathway & 4 & 2.30 & 0.05179 & 4.731 \\
\hline & & Carboxylic acid metabolism & 9 & 5.17 & 0.05512 & 2.163 \\
\hline & & Ubiquitin cycle & 9 & 5.17 & 0.06438 & 2.092 \\
\hline & & Lipid metabolism & 10 & 5.75 & 0.07580 & 1.912 \\
\hline & & Intracellular signaling cascade & 15 & 8.62 & 0.08165 & 1.597 \\
\hline & \multirow[t]{5}{*}{405 (84) } & Intracellular signaling cascade & 11 & 13.25 & 0.01106 & 2.462 \\
\hline & & Regulation of transcription & 17 & 20.48 & 0.01421 & 1.841 \\
\hline & & $\begin{array}{l}\text { Negative regulation of nucleobase, nucleoside, nucleotide } \\
\text { and nucleic acid metabolism }\end{array}$ & 4 & 4.82 & 0.03354 & 5.573 \\
\hline & & Negative regulation of cellular metabolism & 4 & 4.82 & 0.04624 & 4.900 \\
\hline & & Protein ubiquitination & 4 & 4.82 & 0.07009 & 4.123 \\
\hline
\end{tabular}


nogenic assay to determine the radiation sensitivity (data not shown). As previously reported (Nishizaki et al., 2001), H1299 showed higher clonogenic survival upon ionizing radiation. Using these two lung cancer cells, we examined the transcriptomes of each cell at 0 , 2, 4, 8, 12 and 24 hours after the exposure using

Table 3. Gene ontology analysis on the genes RR clusters

\begin{tabular}{|c|c|c|c|c|c|c|}
\hline $\begin{array}{l}\text { Response } \\
\text { pattern }\end{array}$ & $\begin{array}{c}\text { Cluster } \\
\text { (gene No.) }\end{array}$ & GO term & Count & $\%$ & $\mathrm{p}$ value & $\begin{array}{l}\text { Fold } \\
\text { change }\end{array}$ \\
\hline \multirow[t]{20}{*}{ RRE } & \multirow[t]{7}{*}{$501(504)$} & Regulation of metabolism & 85 & 17.71 & 4.09E-05 & 1.522 \\
\hline & & Transcription, DNA-dependent & 74 & 15.42 & $1.90 \mathrm{E}-04$ & 1.515 \\
\hline & & Vesicle-mediated transport & 20 & 4.17 & 4.35E-04 & 2.503 \\
\hline & & Cell cycle & 29 & 6.04 & 0.00205 & 1.852 \\
\hline & & Ubiquitin cycle & 23 & 4.79 & 0.00207 & 2.046 \\
\hline & & Chromosome organization and biogenesis & 15 & 3.12 & 0.00762 & 2.234 \\
\hline & & Protein amino acid phosphorylation & 24 & 5.00 & 0.01227 & 1.726 \\
\hline & \multirow[t]{4}{*}{$502(214)$} & Cell cycle & 17 & 8.17 & $9.46 \mathrm{E}-04$ & 2.549 \\
\hline & & Vesicle-mediated transport & 11 & 5.29 & 0.00211 & 3.232 \\
\hline & & Protein kinase cascade & 8 & 3.85 & 0.01596 & 3.043 \\
\hline & & $\begin{array}{l}\text { Positive regulation of I-kappaB } \\
\text { kinase/NF-kappaB cascade }\end{array}$ & 4 & 1.92 & 0.03703 & 5.421 \\
\hline & \multirow[t]{6}{*}{$503(267)$} & $\begin{array}{l}\text { Nucleobase, nucleoside, nucleotide } \\
\text { and nucleic acid metabolism }\end{array}$ & 74 & 28.35 & 3.51E-08 & 1.803 \\
\hline & & Ubiquitin cycle & 17 & 6.51 & 2.67E-04 & 2.883 \\
\hline & & Response to DNA damage stimulus & 11 & 4.21 & $7.50 \mathrm{E}-04$ & 3.723 \\
\hline & & Protein localization & 17 & 6.51 & 0.00134 & 2.481 \\
\hline & & Establishment of cellular localization & 17 & 6.51 & 0.00175 & 2.417 \\
\hline & & Cell cycle & 17 & 6.51 & 0.00784 & 2.069 \\
\hline & \multirow[t]{3}{*}{504 (190) } & Protein transport & 15 & 8.15 & $1.22 \mathrm{E}-04$ & 3.389 \\
\hline & & Ubiquitin cycle & 13 & 7.07 & $6.95 \mathrm{E}-04$ & 3.203 \\
\hline & & Phosphatidylinositol biosynthesis & 2 & 1.09 & 0.04564 & 42.624 \\
\hline \multirow[t]{15}{*}{$\mathrm{RRC}$} & \multirow[t]{7}{*}{$301(123)$} & $\mathrm{M}$ phase & 13 & 11.30 & $1.45 \mathrm{E}-09$ & 11.219 \\
\hline & & Chromatin assembly or disassembly & 10 & 8.70 & 1.10E-07 & 12.231 \\
\hline & & Response to DNA damage stimulus & 11 & 9.57 & 2.61E-06 & 7.193 \\
\hline & & Cell cycle & 17 & 14.78 & $3.57 \mathrm{E}-06$ & 3.998 \\
\hline & & Response to endogenous stimulus & 11 & 9.57 & 4.59E-06 & 6.751 \\
\hline & & Second-messenger-mediated signaling & 7 & 6.09 & $9.55 \mathrm{E}-04$ & 6.103 \\
\hline & & $\begin{array}{l}\text { Regulation of transcription from RNA } \\
\text { polymerase II promoter }\end{array}$ & 6 & 5.22 & 0.02329 & 3.656 \\
\hline & \multirow[t]{8}{*}{401 (176) } & M phase & 15 & 9.15 & 1.12E-09 & 8.970 \\
\hline & & Organelle organization and biogenesis & 20 & 12.20 & $9.38 \mathrm{E}-05$ & 2.759 \\
\hline & & Transcription from RNA polymerase II promoter & 11 & 6.71 & 0.00868 & 2.634 \\
\hline & & DNA repair & 7 & 4.27 & 0.01457 & 3.509 \\
\hline & & Chromosome organization and biogenesis & 8 & 4.88 & 0.01594 & 3.040 \\
\hline & & Regulation of protein kinase activity & 5 & 3.05 & 0.02793 & 4.331 \\
\hline & & Response to endogenous stimulus & 7 & 4.27 & 0.02978 & 2.977 \\
\hline & & Cell proliferation & 10 & 6.10 & 0.04077 & 2.162 \\
\hline \multirow[t]{7}{*}{$\mathrm{RRL}$} & \multirow[t]{7}{*}{$201(70)$} & $\begin{array}{l}\text { Establishment and/or maintenance of } \\
\text { chromatin architecture }\end{array}$ & 7 & 10.77 & $1.76 \mathrm{E}-04$ & 8.220 \\
\hline & & Cell cycle & 10 & 15.38 & $6.70 \mathrm{E}-04$ & 3.979 \\
\hline & & Regulation of transcription, DNA-dependent & 16 & 24.62 & 0.00502 & 2.104 \\
\hline & & Organelle organization and biogenesis & 9 & 13.85 & 0.00782 & 3.032 \\
\hline & & Regulation of cellular metabolism & 16 & 24.62 & 0.01734 & 1.835 \\
\hline & & Apoptosis & 6 & 9.23 & 0.04261 & 3.055 \\
\hline & & DNA repair & 4 & 6.15 & 0.04591 & 4.897 \\
\hline
\end{tabular}


microarray. Because two lung cancer cells originated from two different individuals with different genetic backgrounds, there should be lots of discrepancy in transcription profiles. However, if we can compare the time-series expression patterns in parallel, it would be possible to detect the genes related to differential responses to ionizing radiation.

Differentially expressed genes (DEGs) were selected by two-way ANOVA on two time-series data $(p<0.05)$.
Then these 2863 DEGs were further classified into 21 clusters according to their expression patterns using SOM (Fig. 1). Fourteen out of 21 clusters containing more than 10 genes could be divided into two groups like RS and RR (Table 1). The level of transcripts in RS clusters are higher in radio-sensitive $\mathrm{H} 460$ cells than in H1299 cells, while RR cluster genes are expressed at higher levels in $\mathrm{H} 1299$ cells. RS group contained 7 clusters with 1272 genes, which were up-regulated in $\mathrm{H} 460$

Table 4. Pathway analysis on DEGs in two-way ANOVA analysis

\begin{tabular}{|c|c|c|c|c|c|}
\hline $\begin{array}{l}\text { Response } \\
\text { pattern }\end{array}$ & Cluster & Pathway term & Count & $\%$ & $\mathrm{p}$ value \\
\hline \multirow[t]{9}{*}{$\mathrm{RSC}$} & \multirow[t]{9}{*}{105} & HSA00330:Arginine and proline metabolism & 8 & 1.60 & 0.0079 \\
\hline & & HSA04010:MAPK signaling pathway & 19 & 3.80 & 0.0322 \\
\hline & & HSA05060:Prion disease & 4 & 0.80 & 0.0459 \\
\hline & & HSA04610:Complement and coagulation cascades & 7 & 1.40 & 0.0613 \\
\hline & & HSA02010:ABC transporters - general & 7 & 1.40 & 0.0083 \\
\hline & & HSA04512:ECM-receptor interaction & 9 & 1.80 & 0.0332 \\
\hline & & HSA00380:Tryptophan metabolism & 8 & 1.60 & 0.0701 \\
\hline & & HSA04210:Apoptosis & 8 & 1.60 & 0.0844 \\
\hline & & HSA04060:Cytokine-cytokine receptor interaction & 18 & 3.60 & 0.0308 \\
\hline RSE & 103 & HSA04060:Cytokine-cytokine receptor interaction & 6 & 4.76 & 0.0117 \\
\hline \multirow[t]{5}{*}{$\mathrm{RSL}$} & \multirow[t]{2}{*}{305} & HSA00120:Bile acid biosynthesis & 3 & 1.72 & 0.0591 \\
\hline & & HSA00520:Nucleotide sugars metabolism & 3 & 1.72 & 0.0119 \\
\hline & \multirow[t]{3}{*}{205} & HSA04910:Insulin signaling pathway & 6 & 3.47 & 0.0658 \\
\hline & & HSA00600:Glycosphingolipid metabolism & 5 & 2.89 & 0.0045 \\
\hline & & HSA00512:O-glycan biosynthesis & 3 & 1.73 & 0.0778 \\
\hline \multirow[t]{16}{*}{ RRE } & \multirow[t]{5}{*}{502} & HSA00280:Valine, leucine and isoleucine degradation & 5 & 2.40 & 0.0011 \\
\hline & & HSA04350:TGF-beta signaling pathway & 4 & 1.92 & 0.0327 \\
\hline & & HSA00072:Synthesis and degradation of ketone bodies & 2 & 0.96 & 0.0640 \\
\hline & & HSA00650:Butanoate metabolism & 4 & 1.92 & 0.0100 \\
\hline & & HSA04510:Focal adhesion & 6 & 2.88 & 0.0305 \\
\hline & \multirow[t]{11}{*}{501} & HSA04620:Toll-like receptor signaling pathway & 6 & 1.25 & 0.0614 \\
\hline & & HSA00770:Pantothenate and CoA biosynthesis & 3 & 0.62 & 0.0986 \\
\hline & & HSA04930:Type II diabetes mellitus & 4 & 0.83 & 0.0816 \\
\hline & & HSA04360:Axon guidance & 7 & 1.46 & 0.0926 \\
\hline & & HSA04910:Insulin signaling pathway & 10 & 2.08 & 0.0038 \\
\hline & & HSA04210:Apoptosis & 7 & 1.46 & 0.0167 \\
\hline & & HSA04120:Ubiquitin mediated proteolysis & 4 & 0.83 & 0.0775 \\
\hline & & HSA04662:B cell receptor signaling pathway & 6 & 1.25 & 0.0179 \\
\hline & & HSA04660:T cell receptor signaling pathway & 6 & 1.25 & 0.0637 \\
\hline & & HSA04650:Natural killer cell mediated cytotoxicity & 8 & 1.67 & 0.0266 \\
\hline & & HSA04070:Phosphatidylinositol signaling system & 7 & 1.46 & 0.0211 \\
\hline \multirow[t]{6}{*}{$\mathrm{RRC}$} & \multirow[t]{2}{*}{401} & HSA04512:ECM-receptor interaction & 4 & 2.44 & 0.0619 \\
\hline & & HSA04110:Cell cycle & 6 & 3.66 & 0.0048 \\
\hline & \multirow[t]{4}{*}{301} & HSA01510:Neurodegenerative disorders & 3 & 2.61 & 0.0330 \\
\hline & & HSA04310:WNT signaling pathway & 5 & 4.35 & 0.0339 \\
\hline & & HSA04120:Ubiquitin mediated proteolysis & 3 & 2.61 & 0.0522 \\
\hline & & HSA04110:Cell cycle & 7 & 6.09 & 0.0003 \\
\hline \multirow[t]{2}{*}{$\mathrm{RRL}$} & \multirow[t]{2}{*}{201} & HSA04010:MAPK signaling pathway & 4 & 6.15 & 0.0743 \\
\hline & & HSA04110:Cell cycle & 3 & 4.62 & 0.0617 \\
\hline
\end{tabular}


cells. When we examine the responses in H460 cells in detail, RS group could be classified into three classes according to the gene expression patterns like constitutive (RSC), early (RSE) and late (RSL) up-regulation by ionizing radiation. RR group also could be classified as $R R C$, RRE and RRL, too.

We have characterized each clusters according to their GO terms over-represented in each cluster significantly $(p<0.05)$. As listed in Table 2 , RS clusters were enriched with $\mathrm{GO}$ terms related to the response to radiation, signal transduction, apoptosis and metabolism. For RR clusters, we could find the GO terms related to cell cycle, DNA damage and apoptosis (Table 3). Using DAVID web-accessible program, we examined the KEGG pathways related to each cluster to understand the time-series data on the cellular responses to ionizing radiation (Table 4). As GO analysis showed the enrichment of terms on biological process, apoptosis

Table 5. Apoptosis-related genes in three different clusters

\begin{tabular}{|c|c|c|}
\hline $\begin{array}{l}\text { Response pattern } \\
\text { (cluster) }\end{array}$ & GenBank accession & Gene name \\
\hline \multirow[t]{26}{*}{ RSC (105) } & NM_001621 & Aryl hydrocarbon receptor \\
\hline & NM_032977 & Caspase 10 , apoptosis-related cysteine peptidase \\
\hline & NM_001228 & Caspase 8 , apoptosis-related cysteine peptidase \\
\hline & NM_001831 & Clusterin \\
\hline & NM_014800 & Engulfment and cell motility 1 \\
\hline & NM_003608 & G protein-coupled receptor 65 \\
\hline & NM_005347 & Heat shock $70 \mathrm{kda}$ protein 5 (glucose-regulated protein, $78 \mathrm{kda}$ ) \\
\hline & NM_006410 & Hiv-1 tat interactive protein $2,30 \mathrm{kda}$ \\
\hline & NM_000875 & Insulin-like growth factor 1 receptor \\
\hline & NM_005531 & Interferon, gamma-inducible protein 16 \\
\hline & NM_000575 & Interleukin 1 , alpha \\
\hline & NM_000576 & Interleukin 1 , beta \\
\hline & NM_000600 & Interleukin 6 (interferon, beta 2) \\
\hline & NM_000314 & Phosphatase and tensin homolog (mutated in multiple advanced cancers 1) \\
\hline & NM_017542 & Pogo transposable element with krab domain \\
\hline & NM_005505 & Scavenger receptor class $b$, member 1 \\
\hline & NM_004760 & Serine/threonine kinase 17a (apoptosis-inducing) \\
\hline & NM_002575 & Serpin peptidase inhibitor, clade b (ovalbumin), member 2 \\
\hline & NM_003955 & Suppressor of cytokine signaling 3 \\
\hline & NM_003238 & Transforming growth factor, beta 2 \\
\hline & NM_003844 & Tumor necrosis factor receptor superfamily, member $10 a$ \\
\hline & NM_003842 & Tumor necrosis factor receptor superfamily, member $10 \mathrm{~b}$ \\
\hline & NM_002546 & Tumor necrosis factor receptor superfamily, member 11b (osteoprotegerin) \\
\hline & NM_018647 & Tumor necrosis factor receptor superfamily, member 19 \\
\hline & NM_006290 & Tumor necrosis factor, alpha-induced protein 3 \\
\hline & NM_005157 & V-abl abelson murine leukemia viral oncogene homolog 1 \\
\hline \multirow[t]{6}{*}{ RRL (201) } & NM_001168 & Baculoviral iap repeat-containing 5 (survivin) \\
\hline & NM_001924 & Growth arrest and dna-damage-inducible, alpha \\
\hline & NM_016639 & Tumor necrosis factor receptor superfamily, member $12 a$ \\
\hline & NM_002128 & High-mobility group box 1 \\
\hline & NM_002466 & V-myb myeloblastosis viral oncogene homolog (avian)-like 2 \\
\hline & NM_001065 & Tumor necrosis factor receptor superfamily, member 1a \\
\hline \multirow[t]{7}{*}{ RRE (501) } & NM_006218 & Phosphoinositide-3-kinase, catalytic, alpha polypeptide \\
\hline & NM_002736 & Protein kinase, camp-dependent, regulatory, type ii, beta \\
\hline & NM_007236 & Calcium binding protein p22 \\
\hline & NM_001165 & Baculoviral iap repeat-containing 3 \\
\hline & NM_014602 & Phosphoinositide-3-kinase, regulatory subunit 4, p150 \\
\hline & NM_006219 & Phosphoinositide-3-kinase, catalytic, beta polypeptide \\
\hline & NM_016123 & Interleukin-1 receptor-associated kinase 4 \\
\hline
\end{tabular}


pathways were significantly changed in both of $R S$ (RSC) and RR (RRE) clusters. In addition, metabolic and signaling pathways were selected in RS group, and cell cycle pathway in RR group.

RSC cluster contained 26 apoptosis-related genes (Table 2), which were over-expressed in $\mathrm{H} 460$ cells, but not changed upon ionizing radiation. Caspases (CASP8 and CASP10), interleukins (IL1A, IL1B and IL6), TRAIL receptors (TNFRSF10A and TNFRSF10B) might play an important role in cell death upon ionizing radiation in H460 cells (Table 5). These genes were present in lower levels, and not induced by irradiation in H1299. RRL cluster contained the genes which were up-regulated in $\mathrm{H} 1299$ cells, and induced in $\mathrm{H} 460$ cells after 8 hours of irradiation. Among 6 genes, Survivin (BIRC5) and GADD45A might be related to radio-resistance of $\mathrm{H} 1299$ cells. RRE clusters in RR group contained 7 apoptosis-related genes like PI3K subunits (PIK3CA, PIK3CB and PIK3R4) and IRAK4. Many genes related to apoptosis were selected in two-way ANOVA analysis, and their differential activity to determine the radiation responses could be sorted out by SOM and pathway analysis.

Radiation sensitivity is usually determined by classical clonogenic assay in radiation biology. But it is not easy to establish cell line from the individual patient's specimen and too slow to be used as a routine work. As a result, there is no available clinical method to predict radiation response to radiation therapy. If we can predict individual response rate to radiation therapy, we can modify total dose and fractionation schedule of radiation therapy individually so as to increase therapeutic ratio.

We have examined the gene expression profiles to understand the underlying molecular changes in cells with different radiation sensitivity. From the differentially expressed genes, we can select the biomarkers to discriminate the radio-resistant tumors from radio-sensitive one. Using those genomic biomarkers, we can develop the platform to check the radiation response in radiotherapy patients. Further study is undergoing with patient's tumor specimen.

\section{Acknowledgements}

This work was supported by the Korea Science and Engineering Foundation (KOSEF) grant funded by the Korea government (MOST) to W.Y. Park (2007-03119) and BK21 to W.Y. Park and J.H. Kim.

\section{References}

Achary, M.P., Jaggernauth, W., Gross, E., Alfieri, A., Klinger, H.P., and Vikram, B. (2000). Cell lines from the same cervical carcinoma but with different radiosensitivities exhibit different cDNA microarray patterns of gene expression. Cytogenet Cell Genet. 91, 39-43.

Dennis, G.Jr., Sherman, B.T., Hosack, D.A., Yang, J., Gao, W., Lane, H.C., and Lempicki, R.A. (2003). DAVID: database for annotation, visualization, and integrated discovery. Genome Biol. 4, P3.

Fukuda, K., Sakakura, C., Miyagawa, K., Kuriu, Y., Kin, S., Nakase, Y., Hagiwara, A., Mitsufuji, S., Okazaki, Y., Hayashizaki, Y., and Yamagishi, H. (2004). Differential gene expression profiles of radioresistant oesophageal cancer cell lines established by continuous fractionated irradiation. Br. J. Cancer 91, 1543-1550.

Golub, T.R., Slonim, D.K., Tamayo, P., Huard, C., Gaasenbeek, M., Mesirov, J.P., Coller, H., Loh, M.L., Downing, J.R., Caligiuri, M.A., Bloomfield, C.D., and Lander, E.S. (1999). Molecular classification of cancer: class discovery and class prediction by gene expression monitoring. Science 286, 531-537.

Kim, I.J., Lim, S.B., Kang, H.C., Chang, H.J., Ahn, S.A., Park, H.W., Jang, S.G., Park, J.H., Kim, D.Y., Jung, K.H., Choi, H.S., Jeong, S.Y., Sohn, D.K., Kim, D.W., and Park, J.G. (2007). Microarray gene expression profiling for predicting complete response to preoperative chemoradiotherapy in patients with advanced rectal cancer. Dis. Colon. Rectum. 50, 1342-1353.

Klopp, A.H., and Eifel, P.J. (2006). Gene expression profiling in cervical cancer: state of the art and future directions. Cancer J. 12, 170-174.

Lee, M.S., Jun, D.H., Hwang, C.I., Park, S.S., Kang, J.J., Park, H.S., Kim, J., Kim, J.H., Seo, J.S., and Park, W.Y. (2006). Selection of neural differentiation-specific genes by comparing profiles of random differentiation. Stem Cells 24, 1946-1955.

Lossos, I.S., Czerwinski, D.K., Alizadeh, A.A., Wechser, M.A., Tibshirani, R., Botstein, D., and Levy, R. (2004). Prediction of survival in diffuse large-B-cell lymphoma based on the expression of six genes. N. Engl. J. Med. 350, 1828-1837.

Nishizaki, M., Meyn, R.E., Levy, L.B., Atkinson, E.N., White, R.A., Roth, J.A., and Ji, L. (2001). Synergistic inhibition of human lung cancer cell growth by adenovirus-mediated wild-type p53 gene transfer in combination with docetaxel and radiation therapeutics in vitro and in vivo. Clin. Cancer Res. 7, 2887-2897.

Park, W.Y., Hwang, C.I., Im, C.N., Kang, M.J., Woo, J.H., Kim, J.H., Kim, Y.S., Kim, H., Kim, K.A., Yu, H.J., Lee, S.J., Lee, Y.S., and Seo, J.S. (2002). Identification of radiation-specific responses from gene expression profile. Oncogene 21, 8521-8528.

Pomeroy, S.L., Tamayo, P., Gaasenbeek, M., Sturla, L.M., Angelo, M., McLaughlin, M.E., Kim, J.Y., Goumnerova, L.C., Black, P.M., Lau, C., Allen, J.C., Zagzag, D., Olson, J.M., Curran, T., Wetmore, C., Biegel, J.A., Poggio, T., Mukherjee, S., Rifkin, R., Califano, A., Stolovitzky, G., Louis, D.N., Mesirov, J.P., Lander, E.S., and Golub, T.R. (2002). Prediction of central nervous system embryonal tumour outcome based on gene expression. Nature 415 , 436-442.

Snyder, A.R., and Morgan, W.F. (2004). Gene expression profiling after irradiation: clues to understanding acute 
and persistent responses? Cancer Metastasis Rev. 23, 259-268.

Tewari, D., Monk, B.J., Al-Ghazi, M.S., Parker, R., Heck, J.D., Burger, R.A., and Fruehauf, J.P. (2005). Gene expression profiling of in vitro radiation resistance in cervical carcinoma: a feasibility study. Gynecol. Oncol. 99, 84-91.

Weidhaas, J.B., Babar, I., Nallur, S.M., Trang, P., Roush, S., Boehm, M., Gillespie, E., and Slack, F.J. (2007). MicroRNAs as potential agents to alter resistance to cy- totoxic anticancer therapy. Cancer Res. 67, 11111-11116. Wong, Y.F., Sahota, D.S., Cheung, T.H., Lo, K.W., Yim, S.F., Chung, T.K., Chang, A.M., and Smith, D.I. (2006). Gene expression pattern associated with radiotherapy sensitivity in cervical cancer. Cancer J. 12, 189-193.

Wu, H.G., Bang, Y.J., Choi, E.K., Ahn, Y.C., Kim, Y.W., Lim, T.H., Suh, C., Park, K., and Park, C.I. (2002). Phase I study of weekly docetaxel and cisplatin concurrent with thoracic radiotherapy in Stage III non-small-cell lung cancer. Int. J. Radiat. Oncol. Biol. Phys. 52, 75-80. 